\title{
New Packaging Concepts: Bridging Devices and Applications
}

\author{
Shiori Idaka, \\ European Research Co-operation Centre. \\ MITSUBISHI ELECTRIC EUROPE B.V. \\ Ratingen, Germany
}

\begin{abstract}
The performance of power modules has continued to improve with Si IGBTs (Insulated Gate Bipolar Transistors), and current capacities ranging from several A to several thousand A and rated voltages ranging from several hundred $V$ to several thousand $V$ have been realized. However, with the expansion of power electronics applications and the demand for even higher efficiency, the specifications required for power modules are becoming more diverse, with lower loss, smaller size and weight, higher density, environmental resistance, longer life and so on. In addition, WBGs (Wide Band-gap semiconductors) such as SiC and GaN have been commercialized, and innovative approaches to bring out the performance of these devices are expected. In this paper, we will discuss the latest trends and future prospects of packaging technology for power modules in the domain of die bonding, wiring, encapsulation, insulation, and functional integration.
\end{abstract}

Keywords: power module, packaging,

\section{Introduction}

The power modules are at the forefront of the latest energy innovations that seek to solve global environmental issues while creating a more affluent and comfortable society for all. Some of these innovations are photovoltaic (PV) and wind power generation from renewable energy sources, smart grids realizing efficient supply of power, electric vehicles (xEV) that take the next step in reducing carbon emission and fuel consumption, and home appliance that achieve groundbreaking energy savings. Whether in appliances, railcars, $\mathrm{xEV}$ or industrial systems, the power modules are key elements in changing the way energy is used.

As the application of power modules expands, the performance requirements and operating environments for power modules are becoming more and more diverse. In order to achieve these requirements, it is important not only to improve the characteristics of power semiconductor devices, but also to maximize their performance and to protect them from external factors through the use of packaging technology [1].

\section{Power module}

Since power semiconductors cannot be directly installed in equipment, power modules are those that are packaged so that they can be built into equipment. The role of the package is to provide electrical and mechanical interface with systems and protection from external stress.
Figure 1 shows the package structure of a conventional power modules. One is a case-type package, which is mainly used for high-voltage industrial equipment. It consists of insulating substrates and semiconductor chips stacked on a metal base plate via solder, and put into a plastic case. Inside the case, aluminum wires are used to connect the chips to each other, to the substrate and to external terminals. And the inside of the case is filled with silicone gel. They are fixed to the heat sink via a TIM (Thermal Interface Material) such as silicone grease, and the heat generated by the power semiconductor is dispersed through the solder, insulating substrate, base plate, and TIM to the outside by the heat sink. As the semiconductor type, combination, and layout can be changed according to the required specifications, the advantage of them is that it offers a design flexibility.

The other is a transfer molded package, which is used for applications with relatively small power, like home appliances. The structure consists of a semiconductor chip soldered onto a copper lead frame, wired with aluminum wires, and encapsulated with epoxy resin. Then, as with the case-type module, it is fixed to the cooling fins via TIM. Transfer molding technique leads to high productivity.

In either structure, the functions required for a power module are ; bonding the semiconductor chips to an insulating substrate or lead frame, electrically connecting internal and with the external terminals, encapsulating with insulating material, and making a path to release the heat generated by the power semiconductor to the 
outside. In other words, die bonding, wiring, encapsulation, and insulating substrate technologies are the essential packaging technologies for power modules.

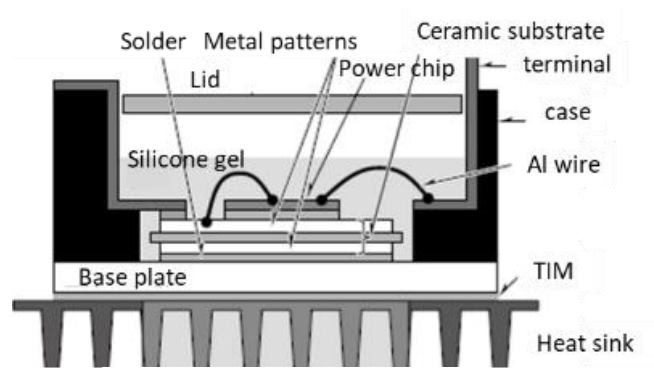

(a) Case-type module

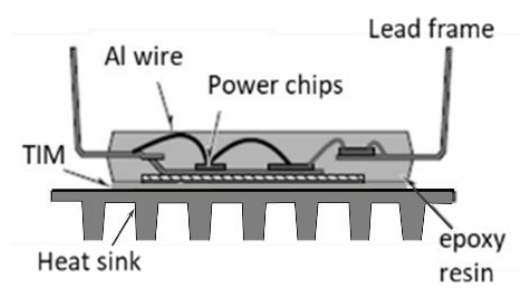

(b) Transfer molded module

Fig.1 Package structure of power modules

\section{Packaging technologies}

\subsection{Die bonding}

In accordance with the RoHS Directive, which restricts the use of lead, tin-based solders are now being mainly used [2]. To achieve the high $\mathrm{Tj}$ operation expected for $\mathrm{SiC}$ devices, sintering technologies using fine metal particles, such as nano-silver, is being adopted [3] [4]. In general, sintering requires high pressure under high temperature, which causes stress on the semiconductor chip (die). Processes to reduce the stress and materials that can be sintered with low pressure are being developed[5].

\subsection{Wiring}

Currently, aluminum or its alloy wire is used for internal connecting between semiconductor chips, to patterns on substrate, and terminals. In order to achieve high current density and high $\mathrm{Tj}$ operation, the use of copper wire has been proposed. However, since the copper wire bonding process requires a large power, it is necessary to form a hard metal layer such as copper or nickel on the semiconductor chip surface as a buffer layer to protect the device structures and chip itself. There are two methods being considered for applying the buffer layer directly by plating, or by attaching a metal plate via sintered materials onto the chip surface [6] [7] [3].

Lead-frame interconnect technology has also been adopted to address high current density. Bonding materials and buffer structures are being considered to protect the device structure on the chip surface from thermal stress[8] [9].

\subsection{Substrate}

Dielectric strength and thermal conductivity as a heat dissipation path are required for the substrate. In general, the substrates are made of ceramics with metal laminated on both sides. Ceramic materials with high strength and high thermal conductivity and methods of applying thick metal patterns are being investigated to overcome the high $\mathrm{Tj}$ operation and thermal stress caused by it [10]. Such ceramic substrates have the problem of warpage in assembly and operation due to the large difference in CTE (Coefficient of expansion) between ceramic and metal patterns. To compensate for this problem, organic/inorganic composite insulating materials with tuned CTE have been commercialized [11].

As another important function, heat dissipation, a structure in which the insulating substrate and cooling fins are integrated has been proposed. This structure eliminates the solder layer and TIM under the substrate, which lowers the thermal resistance and also has the advantage of extending the life of the module [12] [13]. Some examples of new insulating substrate structure concepts are shown in Figure 2. Depending on the application, different properties are considered important. Various approaches have been presented to achieve a balance between performance and cost.

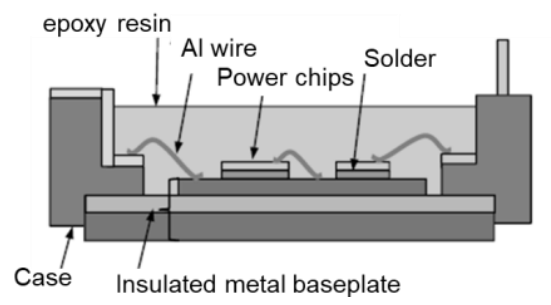

(a) for industry

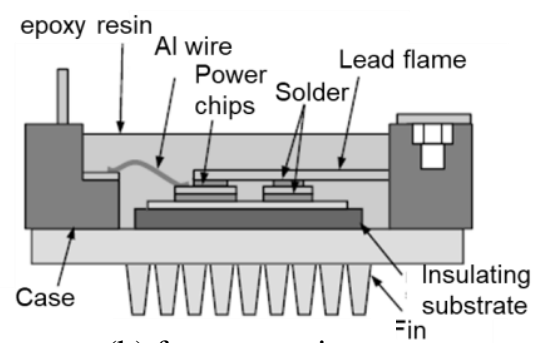

(b) for automotive

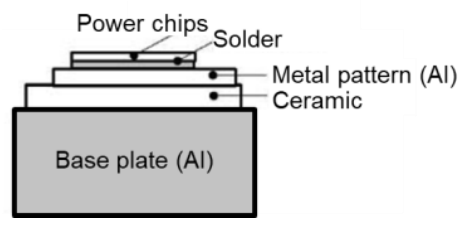

(c) for HV

Fig. 2 New insulating substrate structures 


\subsection{Encapsulation}

The function of the encapsulation is to protect the semiconductor chip and interconnection from electrical and environmental influences. Silicone gels are commonly used in case-type module, but their low molecular compositions make their performance unstable at high temperatures, also has a crystallization point around $-60{ }^{\circ} \mathrm{C}$, which limits its use at high and low temperatures. Their permeability to gas and water limits their use in harsh environments. Epoxy-based resin encapsulation is expected to break through these limitations. Epoxy resins are widely used in transfer molded modules, but their design flexibility is limited as they use a molding die for pressurization. In order to combine the ease-of-process of silicone gel with the robustness of transfer molded resin, thermosetting epoxy resins that can be poured in liquid have been developed [14].

\section{Functional integration}

Integration of functions is required for modules with WBGs and new applications, in addition to the conventional functions of easy of connection and protection from external stresses.

One approach is to "embed" components into the module, such as ICs for driving, various sensors for condition monitoring, and passives for protection and noise reduction. Smaller and more heat-resistant components, and methods of thermal isolation within the module are being considered [15] [16].

Another approach is to "build" the functionality into the module. The integration of sensors and drivers on semiconductor chips has been used. Also, methods of forming passive circuits by applying multilayer circuit technology of PCB and LTCC are being studied [17] [18].

\section{Prospects for the future}

The power electronics systems are expected to have higher functionality, higher efficiency, and wider applications in order to utilize electric energy with less loss. WBGs such as $\mathrm{SiC}$ and $\mathrm{GaN}$ are being promoted as a key to next-generation power electronics. In order to bring out the advantages of these WBGs, not only semiconductor technology but also innovative module technology is required. Therefore, expectations for packaging technology will become more and more important in the future.

\section{REFERENCES}

[1] Gourab, M., Oi, T., Terashima, T., Idaka, S., Nakajima, D. and Goto, Y.: Review of integration trends in power electronics systems and devices, CIPS 2016, (Nuremberg, 2016)

[2] Official journal of the European Union L174/88 https://eur-lex.europa.eu/eli/dir/2011/65/oj
[3] Zhang, Z and Lu, G.-Q.: Pressure-assisted low-temperature sintering of silver paste as an alternative die-attach solution to solder reflow, IEEE Transactions on electronics packaging manufacturing, 25, (2002) , 279-283

[4] Guth, K.: New assembly and interconnect technologies for power modules, CIPS 2012, (Nuremberg, 2012)

[5] Yan, H., Liang, P., Mei, Y. and Feng, Z.: Brief review of silver sinter-bonding processing for packaging hightemperature power devices, Chinese Journal of Electrical Engineering, 6, 2020, issue 3

[6] Haumann, S., Rudzki, J., Osterwald, F., Becker, M. and Eisele, M. : Novel bonding and joining technology for power electronics-Enabler for improved lifetime, reliability, cost and power density, APEC 2013, 622-626

[7] Schmidt, R., König, C. and Prenosil, P. : Novel wire bond material for advanced power module packages, Microelectronics Reliability, vol. 52, no. 9, (2012) 2283-2288

[8] Narazaki, A et al., : Direct beam lead bonding for trench MOSFET \& CSTBT, Proc. ISPSD'05, (Santa Barbara, 2005), 75-78.

[9]. Donlon, J. F, and Motto, E. R., : Transfer molded IGBT module for electric vehicle propulsion, Motor, Drive \& Automation Systems, 2012.

[10] Gurpinar, E., Chowdhury, S. and Ozpineci, B.: , Chinese Journal of Electrical Engineering, 6, 2020, issue 3

[11] Nishimura, T., Mimura, K., Yamamoto, K., Idaka, S., and Shinohara, T.: High heat dissipation and high heat durability technologies for transfer-molded power modules with insulating sheets, Proc. CIPS 2016 (Nuremberg, 2016),

[12] Asada, S., Kondo, S., Kaji, Y. and Yoshida, H.: Resin Encapsulation combined with insulated metal baseplate for improving power module reliability, Proc. PCIM Europe 2016 (Nuremberg, 2016)

[13] Sakai, Y., Oya, D., Iura, S. and Ota, K.: Power cycle lifetime improvement by reducing thermal stress of a new dual HVIGBT module, Proc. EPE'16 ECCE Europe (2016), 1-7

[14] Kaji, Y., Hatanaka, Y., Hiramatsu, S. Kondo, S, Asada, S and Otsubo Y.: Novel IGBT modules with epoxy resin encapsulation and insulating metal baseplate, Proc. ISPSD'16 (Prague, 2016), 457-478

[15] Kempiak, C., Lindemann, A., Thal, E and Idaka, S.: Investigation of the usage of a chip integrated sensor to determine junction temperature during power cycling tests, Proc. CIPS 2018 (Stuttgart, 2018)

[16] Regnat, G., Jeannin, P. O., Ewanchuk, J., Frey, D., Mollov, $\mathrm{S}$ and Ferrieux, J. P.: Optimized power modules for silicon carbide MOSFET, ProcECCE'16 (Milwaukee, 2016)

[17] Feix, G., Hoene, E., Zeiter, O. and Pedersen, K.: Embedded Very Fast Switching Module for SiC Power MOSFETs, Proc. PCIM Europe 2015 (Nuremberg, 2015), 1-7

[18] Weidner, K, Kaspar, $\mathrm{M}$ and Seliger, N.: Planar interconnect technology for power module system integration, Proc. CIPS 2012 (Nuremberg, 2012)

Addresses of the author

Shiori Idaka, Mitsubishi-Electric-Platz 1, 40882 Ratingen, GERMANY, mailto: Shiori.idaka@ meg.mee.com 